neurological outcome was defined as modified Rankin Scale $(\mathrm{mRS}) \leq 2$ within 6 months clinical follow-up.

Results Enrolled patients were categorized into two groups: emergent CAS $(n=27)$ or medical treatment $(n=18)$. Reasons for medical treatment were as follows: spontaneous neurological improvement $(n=4)$, technical failure of emergent CAS $(n=7)$ and good collateral circulation $(n=7)$. Good angiographic outcome (mTICI 3 or $2 \mathrm{~b}$ ) was achieved in 25 (92.6\%) patients of the emCAS group. Reocclusion $(n=3)$ and hyperperfusion syndrome $(n=3$, massive intracerebral hemorrhage, cerebral edema and status epilepticus) were identified after emergent CAS. Hemorrhagic transformation was developed in $11(40.7 \%)$ patients of the emergent CAS group and $3(16.7 \%)$ patients of the medical treatment group $(\mathrm{p}=0.11)$. The emergent CAS group showed a favorable neurological outcome $(51.9 \%$ vs $22.2 \%, \mathrm{p}=0.07)$ and a low rate of recurrent ischemic stroke $(\mathrm{p}=0.01)$ compared to the medical treatment group. In multi-variate analysis, no early neurological deterioration before procedure $(\mathrm{p}=0.04)$, use of IV t-PA $(p=0.03)$,no intracranial tandem lesion $(p=0.02)$ and emergent CAS $(p=0.01)$ were related with a favorable neurological outcome.

Conclusions Emergent CAS for acute ischemic stroke is technically feasible and may give a chance to achieve good neurological outcome. However, physicians should pay attention to the risk of in-stent thrombosis, hemorrhagic transformation and hyperperfusion syndrome.

Disclosures K. Kim: None. K. Jang: None.

\section{E-098 EFFECT OF ENDOVASCULAR REVASCULARIZATION ON NEUTROPHIL-LYMPHOCYTE RATIO AND RELATIONSHIP TO 90 DAY OUTCOME}

${ }^{1} \mathrm{R}$ Abdalla*, ${ }^{1} \mathrm{M}$ Darwish, ${ }^{1} \mathrm{M}$ Aly, ${ }^{2} \mathrm{M}$ Potts, ${ }^{2} \mathrm{~B}$ Jahromi, ${ }^{2} \mathrm{~A}$ Shaibani, ${ }^{2} \mathrm{M}$ Hurley, ${ }^{3} \mathrm{~S}$ Ansari. ${ }^{1}$ Radiology, Northwestern University - Feinberg school of Medicine, Chicago, IL; ${ }^{2}$ Radiology, Neurological Surgery, Northwestern University - Feinberg school of Medicine, Chicago, IL; ${ }^{3}$ Radiology, Neurology, Neurological Surgery, Northwestern University - Feinberg school of Medicine, Chicago, IL

\subsection{6/neurintsurg-2019-SNIS.173}

Purpose Admission Neutrophil-lymphocyte ratios (NLR) is significantly correlated with 90 days functional outcome in acute ischemic strokes. The aim of our study was to detect post thrombectomy changes in NLR over 1 week with various degrees of revascularization and identify a relationship between NLR changes and 90-day functional outcome.

Methods We retrospectively reviewed our prospective stroke database from Nov 2016 to May 2018 for patients who underwent endovascular thrombectomy for anterior circulation large vessel occlusions with an admission NLR (NLR 1) and 72 hours follow-up NLR (NLR 2). We measured stroke severity by NIHSS, degree of recanalization by modified Thrombolysis in Cerebral Infarction (mTICI) score, and clinical outcomes by the modified Rankin Scale (mRS) at 3 months. Univariate analysis was conducted between age, NLR1, NLR2, change in NLR (NLR2-NLR1), NIHSS, mTICI and mRS using correlation coefficient. Change in mean NLR was assessed using Wilcoxon rank sum test. Multivariable logistic regression models were developed to identify effect of NLR 2 on favorable functional outcome (mRS $\leq 2)$ while controlling for age, NIHSS and IV rtPA utilization.

Results 88 patients met our inclusion criteria with a median NIHSS at admission of 18 (4-32), and 90 days mRS of 3 (0-
6). An increase in NLR was identified in $75 \%$ of patients following endovascular thrombectomy. Mean NLR 2 was significantly higher than NLR 1 (5.5 vs $3.1, \mathrm{p}<0.001)$. There was a significantly negative correlation between TICI and change in NLR $(p=0.002)$, and a significantly positive correlation between change in TICI and 90-day mRS $(p=0.034)$, as well as NLR2 and mRS $(p<0.001)$. No correlation was observed between NLR1 and mRS $(p=0.22)$. High NLR2 was an independent predictor of poor functional outcome $(\mathrm{OR}=1.34$, $\mathrm{p}=0.002$ ).

Conclusion NLR is a readily available biomarker that correlates with degrees of revascularization post-thrombectomy. Improved recanalization and reperfusion is associated with lower follow-up NLR at 72 hours and follow up NLR is an independent predictor of functional outcome.

Disclosures R. Abdalla: None. M. Darwish: None. M. Aly: None. M. Potts: None. B. Jahromi: None. A. Shaibani: None. M. Hurley: None. S. Ansari: None.

\section{E-099 HEMICRANIECTOMY RATES FOLLOWING MECHANICAL THROMBECTOMY PRE-MR CLEAN: A RETROSPECTIVE CROSS-SECTIONAL OBSERVATIONAL ANALYSIS}

E Luther*, D McCarthy, R Starke. University of Miami Department of Neurological Surgery, Miami, FL

\subsection{6/neurintsurg-2019-SNIS.174}

Introduction Acute ischemic stroke (AIS) still remains one of the top leading causes of morbidity and mortality in the United States. In 2007, the DESTINY trial demonstrated lower mortality rates in patients with large hemispheric strokes following hemicraniectomy. 7 years later, the MR CLEAN trial showed improved outcomes in AIS following endovascular mechanical thrombectomy (MT) when compared to intravenous tPA alone. However, during the enrollment period for this trial there remains limited data regarding the effects of MT on the need for hemicraniectomy.

Methods A retrospective analysis of the Nationwide Inpatient Sample (NIS) between 1998-2015 was performed. Patients with an ICD-9-CM diagnosis code indicating an anterior circulation ischemic stroke (433.11, 434.01, 434.11, and 434.91) were included. Patients with an ICD-9$\mathrm{CM}$ diagnosis code indicating trauma, neoplasm, SAH/aneurysm, posterior circulation ischemic stroke, or abscess (191.x, 198.3, 324.0, 430, 433.01, 433.21, 437.3, 437.5, 437.6, 443.24, 747.81, 800.xx, 801.xx, 851.xx, 852.xx, 853.xx, and 854.xx) were excluded. In order to limit the analysis to AIS, patients who did not have the ICD-9-CM procedure code of tPA (99.10) were excluded. Those patients who underwent thrombectomy (39.74) and hemicraniectomy (01.24, 01.25, 01.39, 01.53, and 01.59) were also indentified. Logistic regression model was used to analyze the effect of thrombectomy on rates of hemicraniectomy in patients with AIS who received tPA.

Results A total of 59,703 patients in the NIS were identified as having an acute anterior circulation ischemic stroke that subsequently received tPA. 3597 patients were also identified as having undergone MT and 606 patients were found to have undergone hemicraniectomy as well. Logistic regression model demonstrated that patients who underwent MT were significantly more likely to undergo hemicraniectomy (OR 4.52, 95\% CI 3.72-5.49, $\mathrm{p}<0.001)$. 